\title{
Toxoplasmosis congénita, una mirada en la actualidad del tratamiento; revisión de la literatura
}

Congenital toxoplasmosis, a look at contemporary treatment; literature review

Toxoplasmose congênita, um olhar atual no tratamento; revisão de literatura

Yonathan Samuel Rueda-Paez, MD. ${ }^{1}$, Lucas Valbuena-Ruiz, MD. ${ }^{1}$, Nelson Quintero-Pimiento, MD. ${ }^{1}$, Aracely Pinilla-Plata, MD. ${ }^{1}$, Johny Sayago-Silva, MD. ${ }^{1}$

1. Médico general, Universidad Industrial de Santander, Bucaramanga, Santander, Colombia.

Correspondencia: Yonathan Samuel Rueda-Paez, Médico general, Universidad industrial de Santander. Dirección: Cr 34 N 32 -65, apto 1605. Bucaramanga. E-mail: yonathanrueda.med@hotmail.com

Cómo citar: Rueda-Paez YS, Valbuena-Ruiz L, Quintero-Pimiento N, Pinilla-Plata A, Sayago-Silva J. Toxoplasmosis congénita, una mirada en la actualidad del tratamiento; revisión de la literatura. MedUNAB. 2019;22(1):51-63. doi: 10.29375/01237047.2612

\section{INFORMACIÓN ARTÍCULO}

Artículo recibido: 21 de septiembre de 2016

Artículo aceptado: 21 de mayo de 2019

DOI: https://doi.org/10.29375/01237047.2612

\section{RESUMEN}

Introducción. La Toxoplasmosis congénita constituye una causa significativa de morbi-mortalidad neonatal en países de bajos ingresos como Colombia. Puede originar prematuridad, secuelas patológicas y pérdida fetal. El tamizaje en las gestantes y, a su vez, un tratamiento oportuno y adecuado disminuye la transmisión vertical y sus nefastas secuelas. El objetivo es presentar evidencia científica actualizada sobre el tratamiento farmacológico de la Toxoplasmosis Congénita.

Metodología. Se realizó una búsqueda no sistemática en bases de datos: Pubmed, Medline, Clinical Key y Springer. Se incluyeron artículos originales y de revisión de tema publicados desde enero de 2014 hasta abril de 2019.

División de los temas tratados. se abordan la fisiopatología y clínica, el abordaje 
diagnóstico, alternativas de prevención y tratamiento.

Conclusiones. En la actualidad la terapia farmacológica es limitada, los esquemas de manejos se basan en espiramicina o la combinación de sulfadiazina/pirimetamina y ácido folínico; estas moléculas no son del todo bien toleradas y presentan un amplio espectro de reacciones adversas secundario a sus efectos tóxicos; resulta necesario la ejecución de estudios aleatorizados para evaluar su efectividad.

Palabras clave:

Toxoplasmosis Congénita; Sulfadiazina; Pirimetamina; Espiramicina

\section{ABSTRACT}

Introduction. Congenital Toxoplasmosis constitutes a significant cause of neonatal morbimortality in underdeveloped countries like Colombia. It can cause prematurity, pathological after-effects and fetal loss. Screening expectant mothers and in turn, a timely and adequate treatment, reduce vertical transmission and its devastating effects. The objective is to present up-to-date scientific evidence about the pharmacological treatment of Congenital Toxoplasmosis.

Methodology. A non-systematic search of databases was conducted: Pubmed, Medline, Clinical Key and Springer. Original and topic review articles were included dating from January 2014 to April 2019.

Division of topics covered. Physiopathology and clinical pathology, diagnostic approach, prevention and treatment alternatives were addressed.

Conclusions. At this time, pharmacological therapy is limited, management schemes are based on spiramycin or a combination of sulfadiazine/pyrimethamine and folinic acid; these molecules are not very well tolerated and exhibit a wide spectrum of adverse reactions apart from their toxic effects, thus it is necessary to conduct randomized studies to evaluate its effectiveness.

Keywords:

Congenital Toxoplasmosis; Sulfadiazine; Pyrimethamine; Spiramycin

\section{RESUMO}

Introdução. A toxoplasmose congênita é uma causa significativa de morbidade e mortalidade neonatal em países de baixa renda, como a Colômbia. Pode causar prematuridade, sequelas patológicas e perda fetal. A triagem em gestantes e, por sua vez, um tratamento oportuno e adequado diminui a transmissão vertical e suas consequências desastrosas. O objetivo é apresentar evidências científicas atualizadas sobre o tratamento farmacológico da Toxoplasmose Congênita.

Metodologia. Foi realizada uma revisão não sistemática nas bases de dados: Pubmed, Medline, Clinical Key e Springer. Foram incluídos tanto artigos originais, quanto revisões de tópicos publicados de janeiro de 2014 até abril de 2019.

Divisão dos tópicos discutidos. foram abordadas a fisiopatologia e a clínica, a abordagem diagnóstica, alternativas para prevenção e tratamento.

Conclusões. Atualmente, a terapia farmacológica é limitada, os esquemas terapêuticos baseiam-se na espiramicina ou na combinação de sulfadiazina/pirimetamina e ácido folínico; estas moléculas não são totalmente toleradas e apresentam um amplo espectro de reações adversas secundárias aos seus efeitos tóxicos. É necessário realizar estudos randomizados para avaliar sua eficácia.

Palavras-chave:

Toxoplasmose congênita; Sulfadiazina; Pirimetamina; Espiramicina. 


\section{Introducción}

La toxoplasmosis congénita (TC) representa una causa significativa de morbi-mortalidad neonatal en países de bajos ingresos como Colombia (1), a su vez, la incidencia de la enfermedad es marcada en niveles socioeconómicos bajos y vulnerables $(2,3)$.

Existen datos que confirman que las cepas parasitarias en América del Sur son más virulentas comparadas con otros países (1). En Colombia se calcula que entre 2 y 10 de cada mil nacidos vivos padecen de TC (4) y que más de la mitad de mujeres gestantes poseen anticuerpos anti-toxoplasma, por lo tanto se infiere una alta circulación del parásito en la región; sumado a lo anterior, la contaminación del agua con el ooquiste aporta una carga de incidencia de aproximadamente $50 \%$ como lo demuestra el estudio realizado en la región del Quindío en donde se comprobó la presencia de Toxoplasma Ácido Desoxirribonucleico (ADN) en agua potable en el $60 \%$ de las muestras $(5,6)$.

La TC en neonatos puede originar prematurez, alteraciones auditivas, retraso neurológico, coriorretinitis, hidrocefalia, ceguera, entre otros $(7,8)$. Lo anterior se logra prevenir con un adecuado tamizaje durante el control prenatal; sin embargo, en el sistema sanitario colombiano menos del $40 \%$ de las mujeres gestantes son debidamente tamizadas y/o reciben un manejo terapéutico adecuado y/u oportuno para TC $(1,9)$.

El diagnóstico y tratamiento temprano de la toxoplasmosis durante el embarazo ha demostrado en diversos estudios una disminución de la transmisión vertical y de secuelas clínicas (10). El objetivo principal del texto es ofrecer al médico una perspectiva actual del tratamiento farmacológico de la TC basado en la evidencia actual de literatura médico-científica.

\section{Metodología}

Se realizó búsqueda bibliográfica no sistemática en bases de datos: Pubmed, Medline, Clinical Key, y Springer, utilizando los Mesh Term: toxoplasmosis congénita, manifestaciones clínicas, diagnóstico, tratamiento, pirimetamina, sulfadoxina, sulfadiazina, clindamicina. Los criterios de inclusión de los artículos fueron: artículos originales y de revisión de tema publicados desde enero de 2014 hasta abril de 2019. No existieron criterios de exclusión.

\section{División de los temas tratados}

\section{Fisiopatogenia y clínica}

El Toxoplasma gondii (TG) es un parásito protozoario intracelular obligado, que presenta dos fases de ciclo reproductivo: un ciclo sexual por gametogonia en las células epiteliales del intestino delgado de felinos incluidos gatos (hospedero definitivo), y uno asexual en los tejidos extraintestinales de otros huéspedes animales incluido el ser humano (hospederos intermediarios) (11).

El TG cumple su ciclo desde bradizoitos hasta trofozoítos en las células del tracto gastrointestinal desde donde migran y se diseminan por vía sanguínea y/o linfática infectando el miocardio, la retina y el sistema nervioso central, entre otros órganos. Ingresan en las células de forma activa gracias a la producción de hialuronidasas y lisozimas con las cuales atraviesan por microporos las membranas celulares; una vez dentro se multiplican, forman acúmulos citoplasmáticos que provocan lisis celular y reacción inflamatoria que derivan en edema y respuesta inflamatoria mononuclear. Este período de proliferación corresponde a la fase aguda de la toxoplasmosis y es aquí donde el parásito es más vulnerable al tratamiento farmacológico. La posibilidad de afección sistémica severa en el neonato está dada por la virulencia del parásito y su capacidad de migración, así como también por la presencia de variantes alélicas en el huésped siendo la principal de ellas HLA-DQ3 (12-14).

Entre un $25 \%$ y $50 \%$ de los neonatos con TC son prematuros, de los cuales entre el $12 \%$ y el $20 \%$ de estos presenta retraso en el crecimiento intrauterino y bajo peso para edad gestacional. Las manifestaciones clínicas sistémicas son la presencia de alteración ocular, neuropatía, miocarditis, muerte perinatal entre otras manifestaciones que afectan la economía orgánica $(10,11)$.

El globo ocular se ve afectado en varios componentes de su estructura: la retina es el sitio primario de lesión por TC y está presente en el $80 \%$ de los neonatos conmanifestaciones clínicas de la enfermedad. La toxoplasmosis ocular se presenta como una coriorretinitis focal de espesor total, asociada a inflamación granulomatosa necrotizante de la retina con desprendimiento retiniano, coroides, tracto uveal anterior y alteración del humor vítreo, lo cual afecta drásticamente la embriogénesis fisiológica del ojo, que se ve expresada clínicamente en forma de leucocoria. El parásito en forma de taquizoito libre o quiste se encuentra dentro del foco ocular necrótico y la retina adyacente, sitio en el cual también se aprecian infiltrados inflamatorios mononucleares que rodean los vasos sanguíneos de la retina. Es común que el proceso inflamatorio produzca 
alteración del epitelio pigmentario que puede extenderse a la esclerótica adyacente, siendo de forma unilateral o bilateral. Un nido de color blanco esponjoso acompañado de retinitis necrotizante o coriorretinitis adyacente a una cicatriz coriorretiniana es la marca que deja el parásito en el ojo (11-13).

La cámara anterior del ojo es invadida por células inflamatorias y proteínas, que desarrollan grandes y precipitados queratínicos, sinequias y nódulos en el iris, así como formaciones neovasculares en la superficie de este, a veces con aumento de la presión intraocular que genera glaucoma y produce dolor. Los músculos extraoculares se afectan directamente debido a la presencia y toxicidad del parásito, lo cual conlleva a estrabismo, nistagmo, alteraciones visuales y microftalmia. Las lesiones toxoplásmicas pueden afectar la integridad del nervio óptico, así como también las proyecciones visuales del nervio en la corteza cerebral y la corteza visual (15-17).

Las lesiones que ocasiona el $T G$ en el encéfalo son múltiples focos necróticos que aumentan de tamaño, con presencia de taquizoitos asociados a nódulos microgliales. Las manifestaciones se presentan cuando el parásito induce una reacción de afectación vascular de preferencia en zona cortical, ganglios basales y en algunas ocasiones zonas periventriculares. Dicha reacción induce a la formación de zonas necróticas con autolisis que posteriormente pueden calcificarse. (15-17).

La hidrocefalia puede ser la primera manifestación clínica del compromiso encefálico, su aparición obedece a la obstrucción del conducto de Silvio por tejido encefálico que sufrió autolisis y dreno a dicho sitio. Este es el motivo por el cual hasta el 30\% de los neonatos afectados presentan anomalías en el líquido cefalorraquídeo. Una cifra de proteínas $>1 \mathrm{~g} / \mathrm{dl}$ en el Líquido Cefalorraquídeo (LCR) es característica de toxoplasmosis grave del sistema nervioso central y va acompañada habitualmente con hidrocefalia la cual puede requerir manejo quirúrgico a través de la instalación de una válvula de hakim (14-17).

Debido al daño estructural encefálico se presentan episodios convulsivos con patrones muy variados, que incluyen crisis motoras focales, crisis tipo pequeño y gran mal, fasciculaciones y opistótonos. La afectación medular o bulbar puede ponerse de manifiesto por parálisis de las extremidades, dificultad para deglutir, así como también dificultad respiratoria. La microcefalia es otra posible secuela del daño cerebral grave, la cual puede tener distintas manifestaciones clínicas, así como también disfunción cognitiva variable. La TC no tratada durante el primer año de vida, puede causar un deterioro sustancial de la función cognitiva y retraso en el desarrollo (14-17).

En el corazón la presencia de quistes en las fibras miocárdicas se acompaña de cambios patológicos en los puentes contráctiles del músculo cardíaco. Una infiltración focal con los linfocitos, células plasmáticas, células mononucleares y eosinófilos conduce a un estado de inflamación permanente con la consecuente depleción del gasto cardiaco. Áreas focales del miocardio sufren necrosis hialina, fragmentación y calcificación extensa. Las áreas de necrosis se hacen más grandes a medida que la infección progresa y se extienden hacia la periferia, lo que genera contracción asincrónica del músculo cardiaco $(15,18,19)$.

\section{Diagnóstico}

Para el diagnóstico de la $\mathrm{TC}$ es necesario un correcto diligenciamiento de la historia clínica, evaluar aspectos como inmunocompetencia, factores de riesgo, antecedente de pérdidas fetales y correlación con estudios de laboratorio.

Hay 3 escenarios a tener en cuenta, primero la gestante que no adquirió la infección con $T G$ (seronegativa) y que permanece negativa hasta el primer mes después del parto, no hay riesgo de TC. Segundo, gestante con huella serológica que adquirió la infección antes del embarazo, no hay riesgo de $\mathrm{TC}$, a menos que la madre este inmunocomprometida. Tercero, gestante seronegativa que adquirió la infección durante el embarazo o 3 meses antes de la gestación, hay riesgo de TC (20).

Los métodos de laboratorio para diagnóstico de TC se pueden agrupar en dos: directos e indirectos. Los métodos directos incluyen la Reacción en cadena de Polimerasa (PCR), cultivos celulares, tinciones e inmunohistoquímica. Los indirectos abarcan las pruebas serológicas: IgG, Test de avidez, IgM, IgA e IgE.

En Colombia las guías de práctica clínica recomiendan como método de tamizaje de TC en control prenatal las pruebas serológicas; a toda mujer gestante se debe realizar IgG e IgM según su semana de gestación. En el primer trimestre estas pruebas determinan si la gestante presentó en algún momento de su vida contacto con el parásito, adquiriendo así una huella serológica que le da inmunidad durante el embarazo, o por el contrario nunca se expuso al parásito y se encuentra susceptible a adquirir la infección en la gestación. En caso de que la IgM sea reactiva en gestantes menores de 16 semanas se procede a realizar el test de avidez, mientras que, en 
mayores de 16 semanas de gestación, se realiza IgA. La tabla tomada de la guía de toxoplasmosis realizada por la sociedad española de infectología pediátrica maneja algoritmos similares a los nuestros (Tabla 1)(21,22).

En recién nacidos la persistencia de IgG positiva hasta los 12 meses de edad indica infección. Una IgG positiva no significa siempre presencia de la enfermedad, ya que la IgG materna se puede transferir de manera pasiva a través de la placenta al recién nacido, inclusive el recién nacido puede presentar IgM materno hasta los 5 días de vida e IgA materno hasta los 10 días de vida; se debe realizar la prueba de Western blot para diferenciar los anticuerpos de la madre y los del recién nacido; la PCR también es útil para el diagnóstico, y se encuentra en muestras de líquido cefalorraquídeo, sangre periférica y orina (20).

\section{Prevención}

En la prevención es primordial la educación, pues es aquí donde se aprecia un verdadero cambio en la prevención de la infección materna (23); se debe enseñar a las madres sobre las formas de contagio del parásito: la principal vía de transmisión al ser humano es la vía oral, de la ingesta del ooquiste en alimentos contaminados. Los principales factores de riesgo son el consumo de alimentos contaminados, exposición a la carne cruda o mal cocinada, mala higiene de manos, consumo de agua no potable y viajes a áreas endémicas de toxoplasmosis (24). A continuación, se enlista las principales recomendaciones para la prevención de la TC, tomadas de la guía de toxoplasmosis congénita en los Estados Unidos de la American Academy of pediatrics (25):

- La carne de res y cerdo debe ser cocinada al menos a $63{ }^{\circ} \mathrm{C}$ y la carne de aves de corral al menos a 73 ${ }^{\circ} \mathrm{C}$, evitando contacto de las mucosas con la carne cruda.

- Al manipular carne cruda se deben usar guantes y lavarse las manos después de la manipulación.

- Los utensilios y superficie de la de cocina deben lavarse exhaustivamente después del contacto con la carne.

- Evitar consumo de leche no pasteurizada, evitar las ostras crudas, almejas o mejillones; el consumo de frutas y verduras debe estar precedido por un adecuado lavado de estas.

- Evitar el consumo de agua sin tratar (potencialmente infectada por heces de gatos).

- Evitar el contacto con material de jardinería, tierra, arena, en caso de que no se pueda hacer uso de guantes desechables, y posteriormente el respectivo lavado exhaustivo de las manos.
- Los gatos domésticos deben permanecer en el interior, no manipular gatos callejeros, la arenera para gatos debe cambiarse diariamente ya que el TG se convierte en infeccioso de 1 a 5 días después de que se eliminan las heces del gato.

\section{Tratamiento}

Realización del tamizaje preconcepcional y durante la gestación, así como asesoramiento adecuado en medidas higiénicas de prevención en mujeres seronegativas son las principales medidas para evitar la infección fetal, por tal razón es fundamental fortalecer los programas de control preconcepcional y prenatal en todas las mujeres y así clasificar cada gestante según su estado de riesgo (26).

En cuanto a la eficacia del tratamiento se encuentran vacíos en la literatura científica debido a la falta de unanimidad de los resultados. Una revisión de Cochrane de 3,332 estudios publicados en los últimos 30 años concluyó que el tratamiento prenatal en presencia de seroconversión durante el embarazo no disminuye la transmisión vertical pero podría reducir la gravedad en la presentación de toxoplasmosis congénita (27).

Asimismo, el meta-análisis sobre la efectividad del tratamiento a nivel prenatal realizado por el grupo de estudio SYROCOT en 2007 encontró que en gestantes a quienes se les inicia el tratamiento anti-toxoplasmosis dentro de las 3 semanas posterior a la seroconversión la probabilidad de transmisión vertical es de un $52 \%$ menor respecto a las maternas que recibieron el tratamiento tardíamente (28); aunque dicha diferencia no fue epidemiológicamente significativa, en este mismo estudio también se concluyó que el tratamiento prenatal no redujo la gravedad clínica de la enfermedad. Otro estudio, realizado por Hotop A y col., evaluó la eficacia del inicio temprano de tratamiento después de la infección primaria por $T G$ durante el embarazo, en donde se les administró espiramicina a las embarazadas seropositivas hasta la semana 16, seguido de 4 semanas con pirimetamina/sulfadiazina y ácido folínico; los resultados fueron favorables, documentando bajas tasas de transmisión vertical de TC (29).

Con el fin de evitar la transmisión vertical se ha usado la espiramicina en mujeres gestantes desde el momento de la concepción hasta la semana 18 de gestación $(30,31)$, y después de la semana 18 de gestación se recomienda la combinación de pirimetamina/sulfadiazina y ácido folínico, ya que se cree que estos medicamentos al poder atravesar la placenta pueden proporcionar un tratamiento efectivo al feto (32). 
Tabla 1. Diagnóstico indirecto de toxoplasmosis congénita

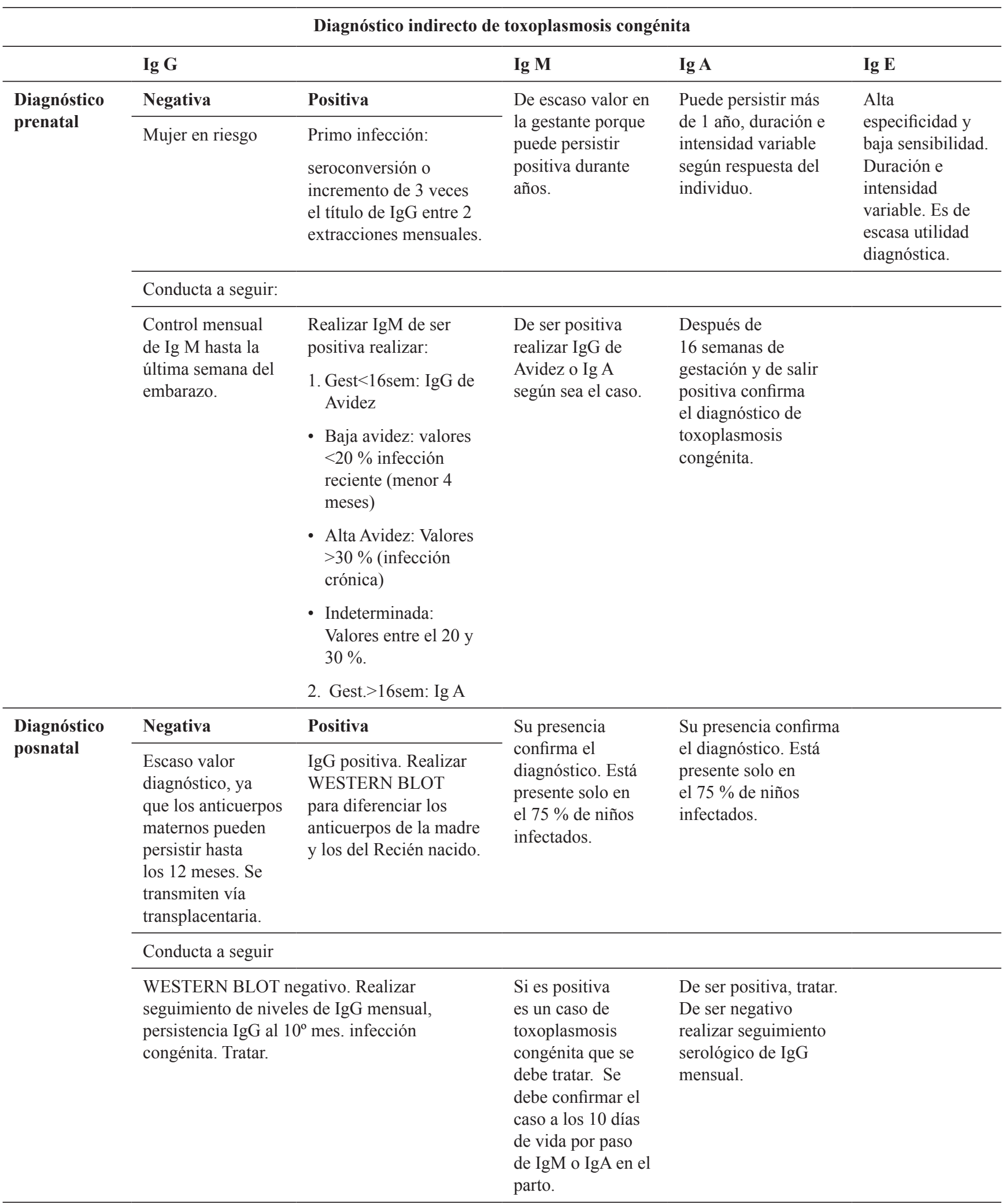

Fuente: Tomado y modificado a partir de Gómez JE, et al. Guía práctica clínica para toxoplasmosis durante el embarazo y toxoplasmosis congénita en Colombia. Infect [Internet]. 2007 [Citado 2 de julio de 2019];11(3):129-141. Recuperado a partir de: http://www.scielo.org.co/scielo.php?script=sci_arttext\&pid=S0123-93922007000300006 
La espiramicina es un macrólido cuyo mecanismo de acción consiste en la inhibición de las síntesis de proteínas mediante la unión a la subunidad $50 \mathrm{~S}$ del ribosoma. Al administrarse oralmente se absorbe de manera incompleta con una biodisponibilidad del $33 \%$ al $39 \%$, la cual se ve afectada si se administra en conjunto con alimentos; la máxima concentración plasmática se logra entre 3 y 4 horas posterior a su administración, alcanza altas concentraciones en pulmones, bronquios, amígdalas, senos nasales y tejido pélvico femenino; sin embargo, la concentración en la sangre fetal es solo del $50 \%$ respecto a la concentración en suero materno, pero las concentraciones alcanzadas a nivel placentario son cinco veces más altas que las obtenidas en el suero y además ha demostrado ser segura para la mujer embarazada y el feto, al contrario de la pirimetamina que no debe ser administrada en el primer trimestre del embarazo por su efecto teratogénico en ratones (33). Por otra parte, la excreción de este medicamento se da principalmente de forma hepática en un $80 \%$ de la dosis administrada y en un porcentaje mínimo de manera renal del $4 \%$ al $14 \%$.

Al ser categoría B en el embarazo y no tener efecto teratogénico, la espiramicina puede ser utilizada como monoterapia para el tratamiento de la TC, la cual reduce la transmisión de esta enfermedad al feto. En la literatura se ha documentado una disminución en la transmisión vertical del $8 \%$ al $25 \%$ en el primer trimestre y del $19 \%$ al $54 \%$ en el tercer trimestre. Cuando existe infección confirmada se recomienda iniciar espiramicina en la dosis de $3 \mathrm{~g} /$ por día vía oral hasta culminar el embarazo; no obstante, en caso de confirmación de la transmisión vertical de TC por pruebas de PCR o ecografías que sugieren compromiso neurológico, se recomienda el cambio a pirimetamina más sulfadiazina $1 \mathrm{mg} / \mathrm{kg} /$ por día y $100 \mathrm{mg} / \mathrm{kg} /$ por día respectivamente, más ácido folínico.

Son escasos los estudios donde se evalúa la eficacia y la seguridad de la espiramicina: dos estudios realizados en Brasil demostraron una disminución en la gravedad clínica de la infección por toxoplasmosis de los pacientes que fueron tratados prenatalmente respecto a los que no fueron tratados; el primero de ellos realizado por Avelino M y col., en el cual se halló que el $18.6 \%$ de los casos que fueron tratados prenatalmente presentaron disminución significativa de infección en relación con los que no fueron tratados en un 60.7 \% (34). El segundo estudio elaborado por Rodríguez I y col. muestra que, en el grupo tratado prenatalmente, el $29.6 \%$ nació con clínica de infección por toxoplasma, aunque no grave, al contrario del grupo no tratado en donde el $68.4 \%$ nació con una infección clínica grave (35).
En la guía de atención integral para la prevención, detección temprana y tratamiento de las complicaciones del embarazo, parto y puerperio: sección toxoplasmosis en el embarazo (10), se recomienda como tratamiento de primera línea la combinación de pirimetaminasulfadiazina y ácido folínico, y como alternativas, ya sea por efectos adversos o limitaciones, la clindamicina, sulfadoxina o azitromicina en conjunto con Pirimetamina más ácido folínico, también se referencia el cotrimoxazol en algunas revisiones (Tabla 2).

La pirimetamina es una diaminopirimidina antipalúdico que tiene como mecanismo de acción inhibir la dihidrofolato reductasa, por lo que reduce los niveles de ácido tetrahidrofólico del parásito, afectando la replicación de $\mathrm{ADN}$; es de absorción lenta pero completa después de la administración oral, al cabo de 2 a 6 horas alcanza las concentraciones plasmáticas máximas, presenta alta unión a proteínas $(90 \%)$, tiene efecto de primer paso, posee gran biodistribución alcanzando inclusive al líquido cefalorraquídeo, la semivida plasmática es larga, de 85 a 100 horas, y se eliminan varios metabolitos por orina y leche materna.

Entre las reacciones adversas se encuentran la anemia megaloblástica que responde a la suspensión de fármaco, arritmias, anorexia, leucopenia, pancitopenia, hematuria, anafilaxia, y en combinación con las sulfonamidas pueden producir reacciones cutáneas como el eritema multiforme, síndrome de Stevens- Johnson, necrólisis epidérmica tóxica, urticaria, dermatitis exfoliativa y hepatitis (36). En el año 2017, Ben R y col. publicaron una revisión sistemática en donde se evaluó el perfil de seguridad de la pirimetamina en las tres principales manifestaciones clínicas de la toxoplasmosis: encefalitis por toxoplasma, toxoplasmosis ocular y $\mathrm{TC}$; se identificaron 13 estudios relacionados con el tratamiento de la TC con pirimetamina, y en 8 de los 13 estudios $(61.5 \%)$ se suspendió o se cambió el tratamiento por eventos adversos. En el $76.9 \%$ de los estudios se informó supresión de la médula ósea en los niños, neutropenia en el $53.8 \%$, anemia $53.8 \%$, trombocitopenia $23.1 \%$ y eosinofilia en el $7.7 \%$, otras reacciones adversas como molestias gastrointestinales se reportó en el $23.1 \%$ de los estudios, reacciones dermatológicas como erupciones en el $30.8 \%$ y reacciones de hipersensibilidad, aumento de bilirrubinas $y$ hepatitis en un $15 \%(37)$.

La sulfadiazina pertenece a la familia de las sulfonamidas, es un bacteriostático cuyo espectro abarca bacterias gram negativas y gram positivas, su mecanismo de acción consiste en inhibir de manera competitiva la dihidropteroato sintasa responsable 
Tabla 2. Tratamiento farmacológico de la toxoplasmosis congénita, farmacocinética, dosis y reacciones adversas

\begin{tabular}{|c|c|c|c|c|c|c|}
\hline Fármaco & $\begin{array}{l}\text { Mecanismo de } \\
\text { acción }\end{array}$ & $\begin{array}{l}\text { Vida } \\
\text { media }\end{array}$ & Excreción & $\begin{array}{l}\text { Unión a } \\
\text { proteínas }\end{array}$ & Dosis & Efectos adversos \\
\hline Pirimetamina & $\begin{array}{l}\text { Inhibición de } \\
\text { la dihidrofolato } \\
\text { reductasa }\end{array}$ & $\begin{array}{l}85 \text { a } 100 \\
\text { horas }\end{array}$ & $\begin{array}{l}\text { Orina } \\
\text { y leche } \\
\text { materna }\end{array}$ & $90 \%$ & \multirow{2}{*}{$\begin{array}{l}\text { Pirimetamina } \\
2 \mathrm{mg} / \mathrm{kg} \text { dosis } \\
\text { inicial y continuar } \\
1 \mathrm{mg} / \mathrm{Kg} \text { por día } \\
\text { hasta completar } \\
\text { un año y la } \\
\text { Sulfadiazina } \\
\text { 100mg/Kg por } \\
\text { día distribuidos } \\
\text { en dos dosis hasta } \\
\text { completar un año, } \\
\text { VO. }\end{array}$} & $\begin{array}{l}\text { Anemia megaloblástica, } \\
\text { arritmias, anorexia, } \\
\text { leucopenia, pancitopenia, } \\
\text { hematuria, anafilaxia y } \\
\text { en combinación con las } \\
\text { sulfonamidas pueden } \\
\text { producir reacciones } \\
\text { cutáneas. }\end{array}$ \\
\hline Sulfadiazina & $\begin{array}{l}\text { Inhibición } \\
\text { de manera } \\
\text { competitiva } \\
\text { de la } \\
\text { dihidropteroato } \\
\text { sintasa }\end{array}$ & 10 horas & $\begin{array}{l}\text { Orina } \\
\text { y leche } \\
\text { materna }\end{array}$ & $55 \%$ & & $\begin{array}{l}\text { Cristaluria, anemia } \\
\text { hemolítica aguda, } \\
\text { agranulocitosis, anemia } \\
\text { aplásica en pacientes } \\
\text { con reserva medular } \\
\text { limitada, anorexia, } \\
\text { náuseas, vómito, fiebre, } \\
\text { kernícterus, reacciones de } \\
\text { hipersensibilidad. }\end{array}$ \\
\hline Sulfadoxina & $\begin{array}{l}\text { Inhibición } \\
\text { de manera } \\
\text { competitiva } \\
\text { de la } \\
\text { dihidropteroato } \\
\text { sintasa }\end{array}$ & $\begin{array}{l}100 \text { a } 230 \\
\text { horas }\end{array}$ & $\begin{array}{l}\text { Orina } \\
\text { y leche } \\
\text { materna }\end{array}$ & $80 \%$ & $\begin{array}{l}\text { ( } 25 \mathrm{mg} \text { de } \\
\text { Pirimetamina } \\
+500 \mathrm{mg} \text { de } \\
\text { sulfadoxina). } \\
\text { Dosis de carga: } \\
50 \mathrm{mg} / \mathrm{kg} \text { según } \\
\text { sulfadoxina el } \\
\text { primer día. Dosis } \\
\text { de mantenimiento: } \\
25 \mathrm{mg} / \mathrm{kg} \text { en dosis } \\
\text { única semanal } \\
\text { hasta el primer año } \\
\text { de vida asociado } \\
\text { a ácido folínico } \\
7,5 \mathrm{mg} / \text { por día }\end{array}$ & $\begin{array}{l}\text { Náuseas, vómito, diarrea, } \\
\text { estomatitis, hepatitis; } \\
\text { trastornos hemáticos: } \\
\text { trombocitopenia } \\
\text { asintomática, leucopenia } \\
\text { y anemia megaloblástica; } \\
\text { alteraciones dermatológicas: } \\
\text { prurito, urticaria, dermatitis } \\
\text { exfoliativa, eritema } \\
\text { multiforme; cefalea, neuritis } \\
\text { periférica, ataxia, vértigo, } \\
\text { tinnitus, convulsiones; } \\
\text { infiltrados pulmonares, } \\
\text { alveolitis alérgica; } \\
\text { insuficiencia renal, nefritis } \\
\text { intersticial, aumento de la } \\
\text { creatinina, y cristaluria. }\end{array}$ \\
\hline $\begin{array}{l}\text { Trimetoprim/ } \\
\text { sulfametoxazol } \\
1: 5\end{array}$ & $\begin{array}{l}\text { Inhiben las } \\
\text { enzimas } \\
\text { secuenciales } \\
\text { que intervienen } \\
\text { en la síntesis } \\
\text { del ácido fólico } \\
\text { bacteriano }\end{array}$ & 13 horas & $\begin{array}{l}\text { Orina } \\
\text { y leche } \\
\text { materna }\end{array}$ & $\begin{array}{l}44 \% \text { para el } \\
\text { trimetoprim } \\
\text { y del } 70 \\
\% \text { para el } \\
\text { sulfametoxazol }\end{array}$ & $\begin{array}{l}\text { 150mg/m2 de TMP } \\
\text { ( } 750 \mathrm{mg} \text { de SMX) } \\
\text { por día, dividida } \\
\text { dos veces al día. }\end{array}$ & $\begin{array}{l}\text { Reacciones de } \\
\text { hipersensibilidad, Anemia } \\
\text { megaloblástica, daño } \\
\text { hepático o renal severos. }\end{array}$ \\
\hline
\end{tabular}




\begin{tabular}{|c|c|c|c|c|c|c|}
\hline Azitromicina & $\begin{array}{l}\text { Inhiben la } \\
\text { síntesis de } \\
\text { proteínas } \\
\text { porque se unen } \\
\text { de manera } \\
\text { reversible a las } \\
\text { subunidades } \\
\text { ribosómicas } \\
50 \mathrm{~S}\end{array}$ & 68 horas & $\begin{array}{l}\text { Orina } \\
\text { y heces } \\
\text { mediante } \\
\text { excreción } \\
\text { biliar }\end{array}$ & $15-30 \%$ & $\begin{array}{l}10 \mathrm{mg} / \mathrm{kg} \text { por día } \\
\text { durante } 8 \text { días, por } \\
\text { dos meses y, luego, } \\
\text { se sigue un mes } \\
\text { con tratamiento } \\
\text { y un mes sin } \\
\text { tratamiento }(50) .\end{array}$ & $\begin{array}{l}\text { Diarrea, náuseas, dolor } \\
\text { abdominal, se ha descrito } \\
\text { hepatitis colestásica, } \\
\text { acúfenos, mareos y pérdida } \\
\text { auditiva. }\end{array}$ \\
\hline Clindamicina & $\begin{array}{l}\text { Ligado } \\
\text { exclusivo a } \\
\text { la subunidad } \\
50 \mathrm{~S} \text { de los } \\
\text { ribosomas } \\
\text { bacterianos } \\
\text { y suprime la } \\
\text { síntesis de } \\
\text { proteína }\end{array}$ & 2.9 horas & $\begin{array}{l}\text { Orina } \\
\text { y heces } \\
\text { mediante } \\
\text { excreción } \\
\text { biliar }\end{array}$ & $90 \%$ & $\begin{array}{l}\text { En niños las dosis } \\
\text { permitidas son } 8- \\
12 \mathrm{mg} / \mathrm{kg} / \text { día. }\end{array}$ & $\begin{array}{l}\text { Cutáneas como erupciones, } \\
\text { eritema multiforme, } \\
\text { Síndrome de Stevens- } \\
\text { Johnson, incremento } \\
\text { irreversible de enzimas } \\
\text { hepáticas, granulocitopenia, } \\
\text { trombocitopenia y } \\
\text { reacciones anafilácticas. } \\
\text { Adicionalmente puede } \\
\text { intensificar el efecto } \\
\text { de los bloqueadores } \\
\text { neuromusculares. }\end{array}$ \\
\hline
\end{tabular}

Fuente: Tomado y modificado a partir de: Goodman A, Gilman A. Las bases farmacológicas de la terapéutica, 13 edición. México Mc Graw Hill. 2018.

de incorporar el ácido paraaminobenzoico (PAVA) en el ácido dihidropteroico precursor de ácido fólico; esta acción es sinergista con el mecanismo de acción de la pirimetamina y el trimetoprim, que tienen como principio inducir bloqueos secuenciales en los trayectos en los que el microorganismo sintetiza tetrahidrofolato.

La sulfadiazina tiene absorción y excreción rápida a diferencia de la sulfadoxina que es de acción prolongada; después de su administración se absorbe en el tubo digestivo, especialmente intestino delgado, se absorbe aproximadamente entre un $70 \%$ y un $100 \%$ de la dosis inicial. La concentración plasmática máxima se logra al cabo de 3 a 6 horas, su semivida plasmática es de 10 horas, con biodistribución amplía a todos los tejidos del cuerpo, incluyendo líquido cefalorraquídeo, y mediana unión a proteínas $(55 \%)$; su excreción es renal en forma libre y acetilada, y se han encontrado concentraciones en leche materna. Las reacciones adversas ocurren en el $5 \%$ de las personas y entre ellas están la cristaluria que se previene con una buena hidratación, anemia hemolítica aguda $(0.05 \%)$, agranulocitosis $(0.1 \%)$, anemia aplásica en pacientes con reserva medular limitada, anorexia, náuseas, vómito, fiebre, kernícterus, reacciones de hipersensibilidad como urticaria, exantema morbiliforme, escarlatiniforme, purpúrico y petequial, eritema nodoso, eritema multiforme, Stevens Johnson, fotosensibilidad, entre otros (36).
Un estudio multicéntrico de la universidad de Chicago realizado entre 1981 y 2004 evaluó la eficacia de la Pirimetamina Sulfadiazina. El estudio tomó 120 niños con $\mathrm{TC}$, a quienes se les administró Pirimetamina $1 \mathrm{mg} / \mathrm{kg}$ en una o dos dosis por día y sulfadiazina $100 \mathrm{mh} / \mathrm{kg} /$ por día repartida en dos dosis poco después del nacimiento hasta completar un año de tratamiento; se efectuó, a su vez, una evaluación inicial y otras periódicas, que determinaban 5 parámetros: anomalías motoras, anomalías cognitivas, deterioro de la visión, nuevas lesiones oculares y pérdida de la audición. Al finalizar el año los resultados fueron favorables en cuanto al desarrollo cognitivo y pruebas auditivas en pacientes sin enfermedad neurológica importante al nacer; del mismo modo, los niños con enfermedad neurológica moderada o grave diagnosticada al nacimiento, a saber, el $72 \%$, presentaron resultados cognitivos normales y el $100 \%$ prueba auditiva normal. Las lesiones oculares de novo solo se presentaron en el $9 \%$ de los niños sin enfermedad neurológica importante y en un $36 \%$ de los niños con enfermedad neurológica moderada o grave. Estos resultados son superiores al compararlos con los niños sin tratar en décadas anteriores $(P<0.01$ para $P<0.001)(38)$.

Las dosis recomendadas son: pirimetamina $2 \mathrm{mg} / \mathrm{kg}$ en dosis inicial y continuar con $1 \mathrm{mg} / \mathrm{Kg}$ por día hasta completar un año, y la sulfadiazina $100 \mathrm{mg} / \mathrm{Kg}$ por día distribuidos en dos dosis hasta completar un año; como la 
sulfadiazina no se encuentra disponible en Colombia se plantean otras opciones que se discuten a continuación.

La sulfadoxina al igual que la sulfadiazina pertenece a la familia de las sulfonamidas y posee el mismo mecanismo de acción inhibitorio de la enzima Dihidropteroato Sintasa; pero que, en comparación con esta, tiene una acción prolongada; se absorbe rápidamente, pero se excretan con lentitud, con una vida media plasmática promedio de 100 a 230 horas ( 7 a 9 días), además tiene una amplia distribución, incluso a LCR, se transporta unido a proteínas en un $80 \%$ y sufre metabolismo hepático (36).

La combinación pirimetamina/sulfadoxina durante muchos años fue el tratamiento de elección para el manejo de la malaria por Plasmodium falciparum no complicado, cuya acción sinérgica de ambos componentes logra destruir las formas parasitarias trofozoítos y esquizontes, sobre todo contra cepas resistentes a cloroquina, donde su mecanismo de acción resulta de la inhibición sucesiva y secuencial de enzimas que interfieren en los dos pasos metabólicos fundamentales en la biosíntesis de folato por el párasito (39).

Existe controversia sobre el uso de esta combinación, debido, en parte, por los efectos adversos graves; asimismo, se plantean dudas sobre los niveles terapéuticos durante la administración. Entre los efectos adversos se encuentran efectos gastrointestinales como náuseas, vómito, diarrea, estomatitis, hepatitis; trastornos hemáticos como trombocitopenia asintomática, leucopenia y anemia megaloblástica; alteraciones dermatológicas como prurito, urticaria, dermatitis exfoliativa, eritema multiforme; trastornos neurológicos como cefalea, neuritis periférica, ataxia, vértigo, tinnitus y convulsiones; trastornos respiratorios como infiltrados pulmonares, alveolitis alérgica; trastornos genitourinarios como insuficiencia renal, nefritis intersticial, aumento de la creatinina, y cristaluria.

En la revisión de literatura, se encontraron dos estudios acerca del fármaco Fansidar ${ }^{\circledR}$; el primero de ellos elaborado por T. Trenque, et al., En este estudio se realizó un análisis farmacocinético de las concentraciones plasmáticas del fármaco mediante un modelo de efectos mixtos no lineal a 89 niños con toxoplasmosis congénita en edades entre 1 semana y 14 años, con pesos que oscilaron entre los $2.9 \mathrm{Kg}$ a los $59 \mathrm{Kg}$, a quienes se les suministró pirimetamina 1.25 $\mathrm{mg} / \mathrm{kg}$ y sulfadoxina $25 \mathrm{mg} / \mathrm{kg} \mathrm{VO}$. Con base en los datos obtenidos concluyeron que el intervalo de administración entre dosis del fármaco debe basarse en el peso corporal o la edad del niño, donde recomiendan como apropiado un intervalo de 7 días para niños menores de 2 años y de 14 días para niños mayores de 2 años (40).
Stephane Corvaisier, et al. realizaron un estudio similar al anterior con una muestra de 32 niños tratados con pirimetamina $1.25 \mathrm{mg} / \mathrm{kg}$ y sulfadoxina $25 \mathrm{mg} / \mathrm{kg}$ por vía oral cada 10 días por 1 año, allí se observó gran variabilidad de concentraciones plasmáticas entre los niños, las cuales diferían entre 8 y 25 veces para la pirimetamina, y de 4 a 5 veces para la sulfadoxina, concluyendo así que las concentraciones séricas son impredecibles incluso cuando la dosis se administra según el peso corporal, por lo que afirman que no se ha logrado establecer una concentración ideal de pirimetamina y sulfadoxina eficiente en niños (41).

Los corticosteroides en combinación con los fármacos antiparasitarios estarían indicados en la toxoplasmosis ocular para disminuir las posibles secuelas de la inflamación intraocular que conllevan al deterioro de la visión, también se indican en presentaciones en las que afecta al sistema nervioso central donde la proteinorraquia sea marcada. En un gran número de estudios se ha propuesto que se requiere realizar ensayos controlados aleatorios, y así determinar el papel de los corticosteroides en dicha patología $(25,42,43)$; sin embargo, la guía de la sociedad española de infectología pediátrica propone el uso de corticoides (prednisona, $1 \mathrm{mg} / \mathrm{kg} /$ por día repartido en 2 dosis), con una duración de 1 a 2 meses en caso de coriorretinitis o hiperproteinorraquia marcada (proteínas en LCR $>1 \mathrm{~g}$ / dl) (21), siendo esta propuesta de gran ayuda al momento de abordar un manejo completo a fin de prevenir complicaciones mayores. En una revisión sistemática de Cochrane Database publicada en el año 2017, se evaluaron los corticosteroides como terapia adyuvante para la toxoplasmosis ocular; los autores concluyeron que se requiere realizar ensayos controlados aleatorios para así determinar el papel de los corticosteroides en dicha patología, y sugieren su uso controlado, de manera individualizada, dependiendo de la gravedad e inmunocompetencia del paciente, ya que el empleo de esteroides puede ser contraproducente (43).

\section{Conclusiones}

La eficacia del tratamiento prenatal de la TC es controvertida, en múltiples estudios no es posible determinar si el tratamiento prenatal previene la transmisión de la infección al feto, dado los diseños de los estudios, número de muestras y aleatoriedad; por tal razón, resulta necesario la ejecución de estudios aleatorizados sobre el tratamiento prenatal de la TC que permitan esclarecer su efectividad; por el contrario, otras investigaciones respaldan una disminución en la gravedad clínica de la infección por toxoplasmosis de 
los recién nacidos que fueron tratados prenatalmente respecto a los que no fueron tratados (24).

Solo se halló un estudio multicéntrico aleatorizado en donde se buscó comparar la eficacia y tolerancia de la pirimetamina (50 $\mathrm{mg}$ qd) más sulfadiazina ( $1 \mathrm{~g}$ tid) versus espiramicina $(1 \mathrm{~g}$ tid) para reducir la transmisión vertical; se realizó en 36 centros franceses, participaron 143 mujeres, a 131 de ellas se le practicó amniocentesis con PCR de $T G$ posterior del tratamiento asignado, y los resultados concluyeron que hubo una menor tendencia de transmisión vertical con pirimetamina más sulfadiazina $(13 / 64-20.3 \%)$ versus espiramicina (7/67 - $10.4 \%$ ); no obstante, estos resultados no son estadísticamente significativos. Igualmente, el grupo que se trató con pirimetamina más sulfadiazina no presentó anomalías cerebrales reportadas ecográficamente; este tipo de estudios promueve la realización de más investigaciones respecto a la profilaxis de la TC (44).

El tratamiento de la TC no es bien tolerado y presenta un amplio espectro de reacciones adversas secundario a sus efectos tóxicos. Esto puede conllevar a la no adherencia del tratamiento; como informó Ben R y col. los eventos adversos más observados en el tratamiento con pirimetamina son a causa de la inhibición del metabolismo de los folatos que se manifiesta en supresión de médula ósea, reacciones dermatológicas y gastrointestinales, por lo que resulta necesario dar un suplemento de ácido folínico en el tiempo de tratamiento se aconseja dar media tableta de $15 \mathrm{mg}$ día intermedio, $y$ en caso de anemia que se administre diariamente (37).

En la actualidad la terapia farmacológica para TC es limitada, el panorama en la creación de nuevas alternativas terapéuticas no es alentador, hay pocos estudios al respecto y escasos fármacos candidatos a ser considerados en el tratamiento de la TC, entre ellos la azitromicina. Esta fue evaluada en el modelo murino por Degerli y col., y dicho fármaco mostró eficacia para inhibir la transmisión vertical de la cepa ME49 de $T G$, sin embargo, para lograr este efecto requirió de altas concentraciones $(45,46)$; otras moléculas empleadas en modelos animales son la atovacuona y diclazurilo. Según informa $\mathrm{Oz}$ y col. la terapia combinada puede ser prometedora $(46,47)$, por ejemplo, el estragol y el timol también fueron probados en el modelo murino anti-Toxoplasma por Oliveira y col., demostrando que las crías nacidas de animales infectados tratados con estas moléculas su peso fue superior al nacimiento en comparación con los controles infectados no tratados (48).

En Colombia el protocolo para el tratamiento de la TC plantea como primera línea de elección la combinación de pirimetamina/sulfadiazina; sin embargo, la sulfadiazina no se encuentra disponible en nuestro país, por tal razón, en la práctica clínica se emplea la combinación de pirimetamina/sulfadoxina. En el artículo alemán publicado en el año 2012 comparan estos dos enfoques terapéuticos, el primero (pirimetamina/ sulfadiazina), basado en el estudio de Toxoplasmosis congénita del National colaborative Chicago, y que es menos tolerado por las gestantes debido a su toxicidad en comparación con el segundo enfoque (pirimetamina/ sulfadoxina) utilizado por un centro de referencia en Toulose Francia, que se asocia a menos toxicidad, además por su prolongada vida provee de un esquema de mayor adherencia (49).

\section{Conflictos de intereses}

Los autores de la presente revisión de tema no presentan ningún conflicto de interés real, potencial o evidente, incluyendo ningún conflicto financiero y/o con terceros.

\section{Referencias}

1. El-Bissati K, Levigne P, Lykins J, et al. Iniciativa global para la toxoplasmosis congénita: un análisis clínico comparativo observacional e internacional. Emergen microbios infecciosos. 2018;7(1):165.

2. Palmezano-Diaz JM, Plazas-Rey LK, Rojas-Carvajal D. Infección por toxoplasma: panorama actual. Spei Domus. 2015;11(22):47-56. Doi: 10.16925/ sp.v11i22.1154

3. Guerina N, Marquez L. La toxoplasmosis congénita: características clínicas y diagnóstico. [Internet]. 2016 [citado 27 de junio de 2019]:1-10. Recuperado a partir de: https://www.uptodate.com/contents/congenital-toxoplasmosis-clinical-features-and-diagnosis

4. Gómez JE, et al. Guía práctica clínica para toxoplasmosis durante el embarazo y toxoplasmosis congénita en Colombia. Infect [Internet]. 2007 [Citado 2 de julio de 2019];11(3):129-141. Recuperado a partir de: http://www.scielo.org.co/scielo.php?script=sci_arttext\&pid=S0123-93922007000300006

5. Gómez-Marin J. Toxoplasma gondii, Brasil. Emerg Infect Dis. 2007;13(3): 512. Doi: 10.3201/ eid1303.060599

6. Karanis P, Aldeyarbi HM, Mirhashemi ME, Khalil KM. El impacto de la transmisión por agua de Toxoplasma gondii y los esfuerzos de análisis para la detección de agua: una descripción general y actualización. Environ Sci Pollut Res Int. 2013;20(1)86-99. Doi: 10.1007/s11356-012-1177-5

7. Botero-Uribe J, Jubiz A, Henao G. Infecciones du- 
rante el embarazo. En Obstetricia y Ginecología. Medellín: CIB fondo editorial. 2015. 313-317p

8. Neu N, Duchon J, Zachariad P. TORCH Infections. Clin Perinatol. 2015;42(1):77-103. Doi: 10.1016/j. clp.2014

9. Gómez-Marin JE, de-la-Torre A, Angel-Muller E, et al. Primer examen multicéntrico en recién nacidos colombianos para la toxoplasmosis congénita.PLoS Negl Trop Dis . 2011; 5 (5): e1195.

10. Cortés JA, Gómez JE, Silva PI, Arévalo L, Arévalo-Rodríguez I, Alvarez MI, et al. Guía de atención integral para la prevención, detección temprana y tratamiento de las complicaciones del embarazo, parto y puerperio: sección toxoplasmosis en el embarazo. Infectio asociación colombiana de infectología [Internet]. 2012 [citado 27 de junio de 2019];16(4):230246. Recuperado a partir de: http://www.scielo.org.co/ pdf/inf/v16n4/v16n4a07.pdf

11. Jameson JL, Fauci AS, Kasper DL, Hauser SL, Longo DL, Loscalzo J. Harrison's principles of internal medicine [Internet]. New York: McGraw-Hill Companies Inc; 2012 [citado 27 de junio de 2019]. 1197-202 p. Recuperado a partir de: https://accessmedicine.mhmedical.com/book.aspx?bookid=2129\#191737008

12. Martín-Hernández II \&Marietta García-Izquierdo SM. Toxoplasmosis en el hombre. BIOQUIMIA [Internet]. 2003 [citado 27 de junio de 2019];28(3):1927. Recuperado a partir de: http://www.redalyc.org/ pdf/576/57611572004.pdf

13. Kliegman RM, Geme J, Schor N. Tratado de pediatría. 2013. 282-295 p.

14. Bollani L, Strocchio L, Stronati M. Congenital toxoplasmosis. Early Human Development. 2013;89:7079. Doi: 10.1016/S0378-3782(13)70107-5

15. Peyron F, Wallon M, Kieffer F, Garweg J. Toxoplasmosis. Remington and Klein's. Infectious Diseases of the Fetus and Newborn Infant. Philadelfia: Elsevier. 2015. 950-1030p.

16. Muñoz-Roldan M, Heimesaat MM, Liesenfeld O. Toxoplasmosis. Manson`s Tropical Diseases. España: Elsevier. 2014. 652-655p. Doi: 10.1016/B978-07020-5101-2.00049-2

17. McAuley JB, Boyer KM, Remington JS, McLeod RL. Toxoplasmosis. Pediatric Infectious Diseases. Massachusetts: Elsevier. 2014. 2986-3010p.

18. Chuang YC, Chen JY, Ji DD, Su PH. Congenital toxoplasmosis in a neonate with significant neurologic manifestations. JFMA. 2012;111(4):230-233. Doi: 10.1016/j.jfma.2011.06.021

19. Macones GA, McNamara J, Wallenstein M, Squires K. Discussion: Congenital toxoplasmosis. Am J Obstet Gynecol. 2010;203(6):e1-3. Doi: 10.1016/j. ajog.2010.09.015

20. Pomares C, Montoya JG. Laboratory Diagno- sis of Congenital Toxoplasmosis. J Clin Microbiol. 2016;54(10):2448-2454. Doi: 10.1128/JCM.00487-16

21. Baquero-Artigaoa F, Del Castillo-Martína F, Fuentes-Corripiob I, Goncé-Mellgrenc A, Fortun-Guaschd C, De la Calle Fernández-Mirandae M, et al. Guía de la Sociedad Española de Infectología Pediátrica para el diagnóstico y tratamiento de la toxoplasmosis congénita. Anales de Pediatría. 2013;79(2):65-132. Doi: 10.1016/j.anpedi.2012.12.001

22. Gomez-Marin JE, Montoya-de-Londondo MT, Castano-Osorio JC. A maternal screening program for congenital toxoplasmosis in Quindio, Colombia and application of mathematical models to estimate incidences using age-stratified data. Am J Trop Med Hyg. 1997;57(2):180-6. Doi: 10.4269/ajtmh.1997.57.180

23. Foulon W, Naessens A, Lauwers S, et al. Impacto de la prevención primaria en la incidencia de toxoplasmosis durante el embarazo . Obstet Gynecol . 1988; 72 ( 3 Pt 1 ): 363-366.

24. Rajapakse S, Weeratunga P, Rodrigo C, de Silva NL, Fernando SD. Profilaxis de la toxoplasmosis humana: una revisión sistemática. Patog Glob Health .2017; 111(7):333-342.

25. Maldonado YA, Read JS. Diagnosis, Treatment, and prevention of congenital Toxoplasmosis in the United States. Pediatrics. 2017;139(2):e1-e53. Doi: 10.1542/ peds.2016-3860.

26. Campello-Porto L, Duarte EC. Association between the risk of congenital toxoplasmosis and the classification of toxoplasmosis in pregnant women and prenatal treatment in Brazil, 1994-2009. Int J Infectio Dis. 2012;16(7):480-6. Doi: 10.1016/j.ijid.2012.01.016

27. Gilbert RE, Harden M, Stanford M. Antibiotics versus control for toxoplasma retinochoroiditis (Review). Cochrane Database of Syst Rev. 2011;(1):1-22. Doi: 10.1002/14651858.CD002218

28. Thiébaut R, Leproust S, Chêne G, Gilbert R; SYROCOT (SystematicReview on Congenital Toxoplasmosis) Study Group. Effectiveness of prenatal treatment for congenital toxoplasmosis: a meta-analysis of individual patients' data. Lancet. 2007;369(9556):115122. Doi: 10.1016/S0140-6736(07)60072-5

29. Hotop A, Hlobil H, Gross U. Efficacy of rapid treatment initiation following primary Toxoplasma gondii infection during pregnancy. Clin Infect Dis. 2012;54(11):1545-1552. Doi: 10.1093/cid/cis234

30. Peyron F, Wallon M, Liou C, Garner P. Treatments for toxoplasmosis in pregnancy. Cochrane Database of Syst Rev. 2000;(2):1-13. Doi: 10.1002/14651858. CD001684

31. Weiss LM, Kim K. Toxoplasma gondii: The model apicomplexan - perspectives and methods. Nueva York: Elsevier.2014.

32. Montoya JG, Remington JS. Management of Toxo- 
plasma gondii infection during pregnancy. Clin Infect Dis. 2008;47(4):554-566. Doi: 10.1086/590149.

33. Daveluy A, Haramburu F, Bricout H, Di Costanzo S, Fourrier A, Tan HK, Gilbert R, Kieffer F, Thiébaut R for the Eurotoxo Group (panel 2). Review of data related to side effects of drugs used in congenital toxoplasmosis [Unpublished report]. Bordeaux (France): The Eurotoxo Group; 2005. Disponible en internet: $\mathrm{http}$ ://eurotoxo.isped.ubordeaux2.fr/WWW PUBLIC/DOC/Side_effects main_drugs_v3.pdf

34. Avelino MM, Amaral WN, Rodrigues IM, Rassi AR, Gomes MB, Costa TL, et al. Programas estatales de toxoplasmosis congénita y atención prenatal. BMC Infect Dis. 2014;14:33. Doi: 10.1186/1471-2334-14-33

35. Rodrigues IM, Costa TL, Avelar JB, et al. Evaluación de los métodos de laboratorio utilizados en el diagnóstico de toxoplasmosis congénita después del tratamiento materno con espiramicina en el embarazo. BMC Infect Dis. 2014; 14.

36. Goodman A, Gilman A. Las bases farmacológicas de la terapéutica, 13 edición. México Mc Graw Hill. 2018.

37. Ben-Harari R, Goodwin E, Casoy J. Adverse Event Profile of Pyrimethamine - Based Therapy in Toxoplasmosis: A Systematic Review. Drugs R D. 2017;17(4):523544. Doi: 10.1007/s40268-017-0206-8.

38. McLeod R, Boyer K, Karrison T, Kasza K, Swisher $\mathrm{C}$, Roizen N, et al. Outcome of treatment for Congenital Toxoplasmosis, 1981-2004: The National Collaborative Chicago Based, Congenital Toxoplasmosis Study. Clin Infect Dis. 2006;42(10):1383-1394. Doi:10.1086/501360

39. Isaza C, Isaza G, Fuentes J, Marulada T, Buritica O, Machado J, et al. Quimioterapia. En Fundamentos de farmacologia en terapeutica(526), 6ta edición. Colombia, Bogota: Celsus. 2014.

40. Trenque T, Simon N, Villena I, Chemla C, Quereux C, Leroux B, et al. Population pharmacokinetics of pyrimethamine and sulfadoxine in children with congenital toxoplasmosis. Br J Clin Pharmacol. 2004;57(6):735741. Doi: 10.1111/j.1365-2125.2004.02077.x.

41. Corvaisier S, Charpiat B, Mounier C, Wallon M, Leboucher G, Al Kurdi M, et al. Population Pharmacokinetics of Pyrimethamine and Sulfadoxine in Children Treated for Congenital Toxoplasmosis. Antimicrob Agents and Chemother. 2004;48(10):3794-3800. Doi: 10.1128/AAC.48.10.3794-3800.2004

42. Adrián-Gutiérrez J, Peñalba-Citores A, Real-Terrón R. Toxoplasmosis congénita: revisión. Acta Pediatr Esp [Internet]. 2006 [Citado 2 de julio de 2019];64(8):372-
376. Recuperado a partir de: http://www.actapediatrica.com/index.php/secciones/revision/649-toxoplasmosis-cong\%C3\%A9nita-revisi\%C3\%B3n\#. XRuC4uhKjIU

43. Jasper S, Vedula SS, John SS, Horo S, Sepah YJ, Nguyen QD.Corticosteroides como terapia adyuvante para la toxoplasmosis ocular.Cochrane Database Syst Rev . 2017;1(1).

44. Mandelbrot L, Kieffer F, Sitta R, Laurichesse-Delmas H, Winer N, Mesnard L, et al. Prenatal therapy with pyrimethamine + sulfadiazine vs spiramycin to reduce placental transmission of toxoplasmosis: a multicenter, randomized trial. Am J Obstet Gynecol. 2018;219(4):386.e1-386.e9. Doi: 10.1016/j. ajog.2018.05.031

45. Degerli K, Kilimcioglu AA, Kurt Ö, Tamay AT, Özbilgin A. Efficacy of azithromycin in a murine toxoplasmosis model, employing a Toxoplasma gondii strain from Turkey. Acta Trop. 2003;88(1):45-50. Doi: 10.1016/S0001-706X(03)00194-3

46. Montazeri M, Sharif M, Sarvi S, Mehrzadi S, Ahmadpour E, Daryani A. Una revisión sistemática de las actividades in vitro e in vivo de los fármacos y compuestos anti- toxoplasma (2006-2016). Microbiol de frente . 2017;8:25.

47. Oz HS. Novel synergistic protective efficacy of atovaquone and diclazuril on fetal-maternal toxoplasmosis. Int J Clin Med. 2014;5(15):921-932. Doi: 10.4236/ ijcm.2014.515124

48. Oliveira CB, Meurer YS, Medeiros TL, Pohlit AM, Silva MV, Mineo TW, et al. Anti-Toxoplasma Activity of Estragole and Thymol in Murine Models of Congenital and Noncongenital Toxoplasmosis. J Parasitol. 2016;102(3):369-76. Doi: 10.1645/15-848

49. Pohl-Gerbutshilfe A, Feiterna-Sperling C, Weizsacker $\mathrm{K}$, Buhrer C. Postnatal therapy for congenital toxoplasmosis: a comparison of 2 different treatment approaches. Z Geburtshilfe Neonatol. 2012;216(2):73-6. Doi: $10.1055 / \mathrm{s}-0032-1308959$

50. Chen EF, Fang YH, Guo DX, Feng XW, Xiang W, Ruan HQ. Follow up of 16 cases with congenital toxoplasmosis treated with azithromiciyn. Zhonghua Er ke Za Zhi [Internet]. 2004 [citado 2 de julio de 2019];42(1):23-5. Recuperado a partir de: https:// www.ncbi.nlm.nih.gov/pubmed/14990100 